\title{
Augmenting Cost-Effectiveness Analysis Will Not Improve Affordability
}

\author{
John B. Watkins, PharmD, MPH, BCPS, and Emily G. Tsiao, PharmD
}

\section{Watkins \& Tsiao's Viewpoint}

1 lthough the quality-adjusted life-year (QALY) is the principal measure of net clinical benefit used by health economists, most would agree that it does not capture the total value that may be gained from a medical intervention. In the previous article, Garrison et et. (2020) present several additional elements of value that could be used to augment the traditional QALY, ${ }^{1}$ based on recent guidance from the ISPOR Special Task Force on Value Assessment Frameworks. ${ }^{2}$ The authors suggest possible ways in which these measures could be included in payers' assessments of new drugs and medical technologies, describe the most effective uncertainty-related novel elements, and discuss what has been or could be done to measure them and aggregate them in a health technology assessment (HTA).

How will knowing the most effective uncertainty-related novel elements and what can be done to measure and aggregate them influence a payer's decision making? Payers look to the HTA community to answer this question. We need guidance on how to use this type of information in order to tackle the very real challenge of affordability in a current state where it is difficult to overcome operational and technological barriers.

The QALY is a limited measure of total net benefit that could be augmented by adding dimensions that may benefit the individual and society. In addition, the coming wave of "curative or transformative" therapies adds a new level of uncertainty to QALY estimates because of extrapolation from relatively shortterm follow-up in clinical trials to expected lifetime horizons. Manufacturers seeking to demonstrate the value of these treatments could justify a higher price by identifying additional value not captured using traditional cost-utility modeling methods. However, this does nothing to alleviate the ongoing affordability problem. Cost-effectiveness must be paired with affordability to be of value to payers.

Of the 9 value dimensions in the ISPOR report that are tied to the individual patient, only 3 are included in the costper-QALY calculation used by the Institute for Clinical and

J Manag Care Spec Pharm. 2020;26(4):407-08

Copyright $\odot 2020$, Academy of Managed Care Pharmacy. All rights reserved.
Economic Review (ICER), National Institute for Health and Care Excellence, Canadian Agency for Drugs and Technologies in Health, and other HTA bodies. One of the 3 dimensions, productivity, is difficult to measure, although it is expected to be present in many cases. Six other values to the individual are suggested. Payers are asked to consider their usefulness in determining coverage policy, 4 of which we examine in this analysis.

First, insurance value has 2 dimensions: financial and physical health. The financial value of insurance is central to its purpose and derives from spreading the risk of financially catastrophic but rare events over a large pool of individuals so that the cost to each is sustainable. Manufacturers of gene therapies justify multimillion dollar prices on the grounds that the system can absorb this cost because of the small patient population, but this assumption fails when the number of treated patients exceeds the ability of the risk pool to absorb the cost. It has been estimated that $10 \%$ of the population has a rare disease. ${ }^{3}$ The National Organization for Rare Disorders (NORD) Rare Disease Database currently lists over 1,200 diseases. ${ }^{4}$ If treatments were available for all of them at current gene therapy prices, the cost would exceed $\$ 50$ trillion!

The physical health benefit of insurance is based on the assumption that proposed treatments would actually be effective. Garrison et al.'s choice of Alzheimer disease as a target example illustrates the problem. Payers will be asked to accept high prices with minimal evidence from relatively short-term studies and a plausible biologic mechanism. Some of these treatments are proposed for asymptomatic individuals to be identified with biomarker tests of uncertain prognostic or predictive value. Individuals might be treated for years with no confirmatory evidence, since it would be impossible to know whether the patient would ever have become symptomatic. Another problem is that, of the plethora of "innovative" treatments brought to market in the last 2 decades, most provide only modest benefit. The huge cost might only produce a slower rate of decline, resulting in a longer period of expensive care, family stress, and loss of productivity by the caregivers. Lifetime high-cost treatment is burdensome to the system even when very effective, as in the case of human immunodeficiency virus suppression. 
Second, by analogy to stock and commodity markets, real option value in health care would derive from the ability of a currently available treatment to extend survival and slow disease progression while, hopefully, medical science finds a cure. Given the progress of today's medical science, this is a realistic argument, but as with the previous sources of value, it is subject to a high degree of uncertainty, making it difficult to include in a calculation.

Related to this is the third value-that is, the value of hope. Patients facing a terminal illness tend to prefer treatments that offer a possible cure, even when the probability is low. This preference will not be reflected in population-level QALY estimates. The systematic review of stated and revealed preferences in cancer treatment by MacLeod et al. (2016) supports this claim. ${ }^{5}$ However, the effect on the overall value calculation appears to be modest.

Garrison et al. also briefly mention the value of knowing, the fourth value. ${ }^{1}$ The value of knowing may be of subjective value to the patient, but insurers have traditionally considered it to be out of the scope of coverage, reimbursing tests only when they are expected to affect treatment decisions.

If sound methodology could be developed to measure some of these elements of value, a rigorous process of factoring them into coverage decisions would require multicriteria decision analysis (MCDA), likely beyond the expertise of most U.S. payers. Developing weights for the different domains in an MCDA would be challenging, as would avoiding methodologic errors such as including interdependent variables, which leads to double counting. Garrison et al. mention this in passing. These difficulties do not necessarily mean we should reject this approach, but unless the modeling is done by a trusted third party, such as ICER, it is unlikely to be useful.

Our most serious concern is that manufacturers will use the incrementally higher value estimates obtained by including additional dimensions of value to justify even higher prices than those that the health care system is currently struggling to bear. It is meaningless to ask whether someone is "willing to pay" what they do not have! Our system simply cannot afford further price increases without realizing offsetting major improvements in efficiency that reduce short-term spending. The lack of public awareness that health care resources in the United States are limited leaves marginal cost out of the political discussion. This must happen if we are to find meaningful solutions.

As noted in Watkins (2018), ${ }^{6}$ Lomas et al. (2018) suggested that if a treatment falls below the willingness-to-pay threshold but does not fit in the budget, we should not say that it is cost-effective but unaffordable, rather, we should say that the threshold is set too high. ${ }^{7}$ In 2015, Claxton et al. published an empirically estimated threshold for the United Kingdom's
National Health Service of $£ 12,936$ per QALY, substantially below the officially stated $£ 20,000-£ 30,000$ threshold. $^{8}$

Expansion of the value framework is a desirable improvement. Considering additional factors may provide a more complete view of the patient experience, enabling decision makers to prioritize treatments that offer the best value, but we must be careful not to jeopardize the financial viability of the system in the process. Payers need and look to the HTA community to provide them with information they can use to operationalize change that makes a difference in the affordability of new innovations for the patient and everyone involved. U.S. payers look to leaders such as ICER to tell us what to do with this information.

\section{Authors}

JOHN B. WATKINS, PharmD, MPH, BCPS, and EMILY G. TSIAO, PharmD, Premera Blue Cross, Mountlake Terrace, Washington.

AUTHOR CORRESPONDENCE: John B. Watkins, PharmD, MPH, BCPS, Premera Blue Cross, 7001 220nd St., S.W., MS 432, Mountlake Terrace, WA 98043. Tel.: 425.918.5146;

E-mail: john.watkins@premera.com.

\section{DISCLOSURES}

No outside funding contributed to this article. The authors are employed by Premera Blue Cross and have nothing to disclose.

\section{REFERENCES}

1. Garrison LP, Zamora B, Li M, Towse A. Augmenting Cost-Effectiveness Analysis for uncertainty: implications for value assessment-rationale and empirical support. J Manag Care Spec Pharm. 2020;26(4):from this issue.

2. Garrison LP, Neumann PJ, Willke RJ, et al. A health economics approach to U.S. value assessment frameworks-summary and recommendations of the ISPOR Special Task Force report [7]. Value Health. 2018;21(2):161-65.

3. Global Genes. RARE facts. Available at: https://globalgenes.org/rare-facts/. Accessed March 2, 2020

4. National Organization for Rare Disorders (NORD). Rare disease database. Available at: https://rarediseases.org/for-patients-and-families/informationresources/rare-disease-information/. Accessed February 19, 2020

5. MacLeod T, Harris A, Mahal A. Stated and revealed preferences for finding new high-cost cancer drugs: a critical review of the evidence from patients, the public and payers. Patient. 2016;9(3):201-22.

6. Watkins JB. Affordability of health care: a global crisis. Value Health. 2018;21(3):280-82

7. Lomas J, Claxton K, Martin S, Soares M. Resolving the "cost-effective but unaffordable" paradox: estimating the health opportunity costs of nonmarginal budget impacts. Value Health. 2018;21(3):266-75.

8. Claxton K, Martin S, Soares M, et al. Methods for the estimation of the National Institute for Health and Care Excellence cost-effectiveness threshold. Health Technol Assess. 2015;19(14):1-503. 